\title{
A direct link between carbohydrate utilization and virulence in the major human pathogen group A Streptococcus
}

\author{
Samuel A. Shelburne III*, David Keith*, Nicola Horstmann ${ }^{\dagger}$, Paul Sumby ${ }^{\ddagger}$, Michael T. Davenport*, Edward A. Graviss ${ }^{\S}$, \\ Richard G. Brennan ${ }^{\dagger}$, and James M. Musser ${ }^{\ddagger \pi}$
}

Departments of *Medicine and §Pathology, Baylor College of Medicine, Houston, TX 77030; ${ }^{\dagger}$ Department of Biochemistry and Molecular Biology, M.D. Anderson Cancer Center, University of Texas, Houston, TX 77030; and ${ }^{\ddagger}$ Center for Molecular and Translational Human Infectious Diseases Research, The Methodist Hospital Research Institute, and Department of Pathology, The Methodist Hospital, Houston, TX 77030

Communicated by Richard M. Krause, National Institutes of Health, Bethesda, MD, December 16, 2007 (received for review October 22, 2007)

\begin{abstract}
Although central to pathogenesis, the molecular mechanisms used by microbes to regulate virulence factor production in specific environments during host-pathogen interaction are poorly defined. Several recent ex vivo and in vivo studies have found that the level of group A Streptococcus (GAS) virulence factor gene transcripts is temporally related to altered expression of genes encoding carbohydrate utilization proteins. These findings stimulated us to analyze the role in pathogenesis of catabolite control protein A (CcpA), a GAS ortholog of a key global regulator of carbohydrate metabolism in Bacillus subtilis. Inasmuch as the genomewide effects of CcpA in a human pathogen are unknown, we analyzed the transcriptome of a $\triangle c c p A$ isogenic mutant strain grown in nutrientrich medium. CcpA influences the transcript levels of many carbohydrate utilization genes and several well characterized GAS virulence factors, including the potent cytolysin streptolysin S. Compared with the wild-type parental strain, the $\triangle$ ccp $A$ isogenic mutant strain was significantly less virulent in a mouse model of invasive infection. Moreover, the isogenic mutant strain was significantly impaired in ability to colonize the mouse oropharynx. When grown in human saliva, a nutrient-limited environment, CcpA influenced production of several key virulence factors not influenced during growth in nutrient-rich medium. Purified recombinant CcpA bound to the promoter region of the gene encoding streptolysin S. Our discovery that GAS virulence and complex carbohydrate utilization are directly linked through CcpA provides enhanced understanding of a mechanism used by a Gram-positive pathogen to modulate virulence factor production in specific environments.
\end{abstract}

ссрA | pharyngitis | regulation | streptolysin | transcriptome

nvestigations in bacterial pathogens have suggested close links between basic metabolic processes and microbial pathogenesis $(1,2)$. For example, bacteria alter transcription of carbohydrate utilization genes and virulence factor production in response to changes in environmental conditions encountered during infection in humans $(3,4)$. Therefore, it is reasonable to speculate that pathogenic bacteria have developed molecular strategies to directly link regulation of carbohydrate utilization and virulence factor production. However, the mechanisms underlying such relationships are largely undefined.

In Bacillus subtilis, alterations in gene transcription in response to environmental carbohydrate concentrations are controlled in part by catabolite control protein A (CcpA), which binds to DNA at catabolite response element (cre) sites (5). Binding of CcpA to cre sites is enhanced by interaction of CcpA with the phosphoprotein HPr-Ser-46-P, the phosphorlyation state of which in turn is affected by uptake of glucose and other readily metabolized carbohydrates by phosphotransferase (PTS) systems (6). Thus, in Bacillus spp., CcpA directly links environmental carbohydrate levels with transcriptional regulation of carbohydrate utilization genes. Most studies of $\mathrm{CcpA}$ have been conducted in
Bacillus spp. (5, 7). Several Gram-positive pathogens encode proteins with significant homology to B. subtilis CcpA and $\mathrm{HPr}$ suggesting that similar molecular processes may occur in other microbes (8-11).

Group A Streptococcus (GAS) causes diverse infections in humans ranging from colonization and uncomplicated pharyngeal and skin infections to necrotizing fasciitis and toxic shock syndrome (12). The diversity in routes and manifestations suggests that GAS colonization and infection involve complex regulatory networks that are differentially regulated in distinct environments (13). In fact, recent genomewide investigations of GAS gene expression have demonstrated that GAS responds to different environments by altering the transcription of genes involved in meeting basic metabolic demands and differential transcription of genes encoding major virulence factors (14-17). These studies have resulted in a new understanding of the relationship between metabolism and virulence in GAS. Here, we report the results of studies that extend this understanding to the molecular level.

\section{Results}

Comparison of GAS Gene Transcript Levels in Saliva and a NutrientRich Medium. Genomewide transcriptome analyses have suggested that differences exist in GAS gene expression during interaction with saliva and the oropharynx compared with growth in laboratory media, but no direct comparison has been done $(15,17)$. We used real-time TaqMan quantitative reverse transcription (QRT) PCR to test the hypothesis that GAS gene transcript levels differ significantly during growth in human saliva, a major component of innate and acquired immunity in the oropharynx, compared with growth in Todd-Hewitt broth with yeast extract (THY). We measured the transcript levels of 78 GAS genes encoding transcription regulators or proteins with either a known or putative extracellular location because of the likelihood such genes are involved in host-pathogen interaction [see supporting information (SI) Table 1]. Fifty-nine of the 78 genes had at least a twofold significantly different transcript level between the two media for at least one of the time points measured (select genes are shown in SI Fig. 7 with gene functions

Author contributions: S.A.S., N.H., P.S., R.G.B., and J.M.M. designed research; S.A.S., D.K., N.H., and M.T.D. performed research; P.S. and J.M.M. contributed new reagents/analytic tools; S.A.S., D.K., N.H., P.S., E.A.G., R.G.B., and J.M.M. analyzed data; and S.A.S., D.K., N.H., P.S., M.T.D., E.A.G., and J.M.M. wrote the paper.

The authors declare no conflict of interest.

Data deposition: Expression microarray data have been deposited in the Gene Expression Omnibus (GEO) database at NCBI, http://www.ncbi.nlm.nih.gov/geo (accession no. GSE10156).

१To whom correspondence should be addressed. E-mail: jmmusser@tmhs.org.

This article contains supporting information online at www.pnas.org/cgi/content/full/ 0711767105/DC1.

() 2008 by The National Academy of Sciences of the USA 
available in SI Table 1). Differences in gene transcript levels between saliva and THY media were especially prominent in the early-exponential growth phase, as 50 genes had significantly different transcript levels at the time point studied. A key finding was that, at the early time point, we observed $\approx 10$-fold increase in the $\operatorname{ccp} A$ transcript level in saliva compared with THY. Together, the data show that GAS markedly alters its transcript profile in response to human saliva and suggest that CcpA mediates some of the observed differences.

Inactivation of the ccpA Gene Results in Medium-Specific Growth Defects. To test the hypothesis that CcpA directly mediates some of the observed transcript differences we created isogenic mutant strain $\Delta c c p A$ from wild-type serotype M1 strain MGAS5005 (confirmatory Southern blot shown in SI Fig. 8). We genetically complemented the $\Delta c c p A$ isogenic mutant strain by using a plasmid capable of replicating in GAS to make strain $\operatorname{comp} \Delta c c p A$. The growth curves for the three strains in THY were superimposable (SI Table 2 and SI Fig. 9). Conversely, compared with wild-type strain MGAS5005, the $\Delta c c p A$ mutant strain had a prolonged lag phase in a glucose medium and an increased (slower) doubling time in a maltose medium (Fig. $9 B$ and $C$ ). Moreover, the $\Delta c c p A$ strain did not reach as high a cell density or maintain as many viable colony-forming units (CFUs) in human saliva as wild-type strain MGAS5005 (Fig. 9D). Gram stain of the $\triangle c c p A$ isogenic mutant strain grown in human saliva did not reveal enhanced clumping or increased chain length, two possibilities to explain the observed CFU differences (data not shown). There was no significant difference in growth between strain MGAS5005 and the $\operatorname{comp} \Delta c c p A$ strain in any of the media tested.

The $\triangle$ ccpA Isogenic Mutant Strain Has an Altered Colony Morphology Associated with Differential Expression of pel/sagA and hasA Transcripts. The $\Delta c c p A$ mutant strain had altered colony morphology and hemolytic phenotype compared with wild-type strain MGAS5005 when grown on blood agar plates (Fig. 1A). The colony diameter $(1.7 \pm 0.1 \mathrm{~mm})$ of the $\Delta c c p A$ strain was significantly less than that of wild-type strain MGAS5005 (2.6 \pm $0.3 \mathrm{~mm}$ ) or the $\operatorname{comp} \Delta c c p A$ strain $(2.4 \pm 0.4 \mathrm{~mm}, P<0.001)$. The observed difference in colony diameter was not caused by a reduced number of bacterial cells, because there was no significant difference in CFUs within colonies of the three strains $\left(1.45 \pm 0.3 \times 10^{9}, 1.21 \pm 0.51 \times 10^{9}, 1.33 \pm 0.49 \times 10^{9}\right.$ for strain MGAS5005, the $\Delta c c p A$ strain, and $\operatorname{comp} \Delta c c p A$ strains, respectively, $P=0.461)$. In addition, the radius of the zone of $\beta$-hemolysis surrounding the $\Delta c c p A$ mutant strain $(1.7 \pm 0.1$ $\mathrm{mm}$ ) was significantly larger than the zone around wild-type strain MGAS5005 $(0.4 \pm 0.1 \mathrm{~mm})$ and the $\operatorname{comp} \Delta c c p A$ strain $(0.6$ $\mathrm{mm} \pm 0.2 \mathrm{~mm}, P=0.005)$.

The $\beta$-hemolytic properties and colony morphology of GAS are determined in part by streptolysin S activity and capsule size, respectively $(18,19)$. Thus, we used real-time TaqMan QRTPCR to test the hypothesis that the $\Delta c c p A$ strain had higher levels of $\operatorname{sag} A$ transcript, the first gene in a nine-gene operon responsible for streptolysin $\mathrm{S}$ production, and lower transcript levels of has $A$, the first gene in a three-gene operon of capsule synthesis genes. Consistent with the hypothesis, the level of $\operatorname{sag} A$ transcript was increased $\approx 20$-fold in the $\Delta c c p A$ mutant strain compared with wild-type strain MGAS5005 (Fig. $1 B$ ). Moreover, the has $A$ transcript was significantly decreased in the $\Delta c c p A$ mutant strain compared with its parental strain at both time points. Although not studied in strain MGAS5005, in another GAS strain the sagA gene is transcribed as part of an RNA molecule with regulatory activity known as the pleiotropic effect locus (pel) (20). Based on QRT-PCR analysis, we found no significant difference in the transcript level of pel and $\operatorname{sag} A$, consistent with the hypothesis that $\operatorname{sag} A$ is part of the larger pel transcript in strain MGAS5005
A
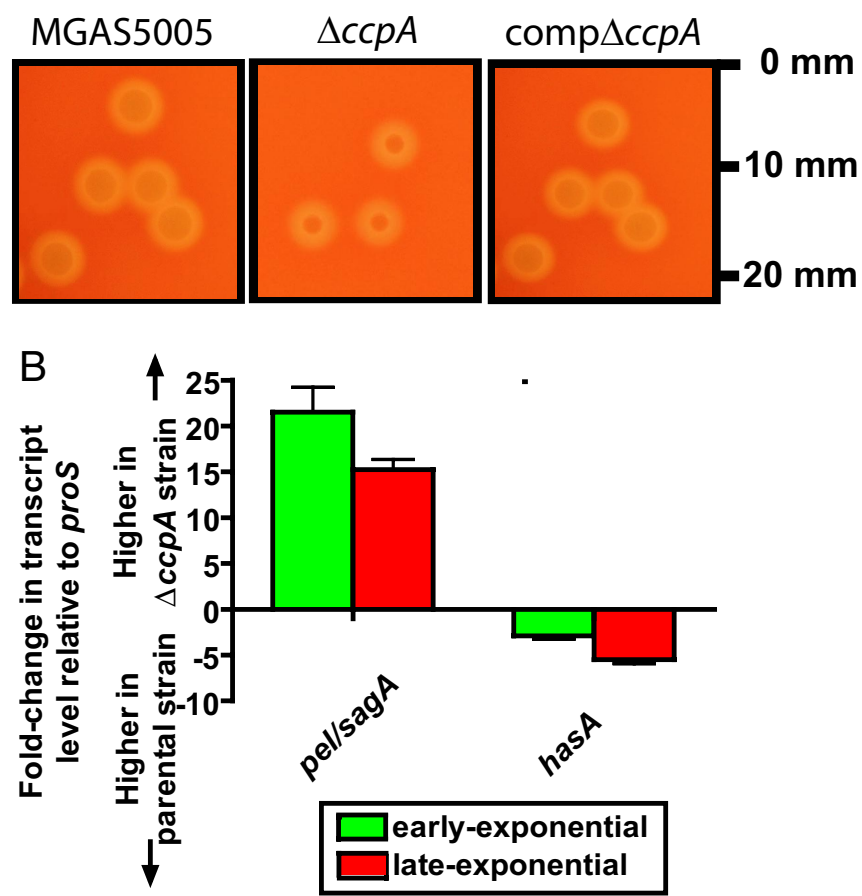

Fig. 1. СсpA influences streptolysins and hyalouronic acid capsule expression. (A) Colony phenotype of strain MGAS5005, its $\Delta c c p A$ mutant derivative strain, and comp $\Delta c c p A$ strain on sheep blood agar plates. The $\Delta c c p A$ strain has significantly increased hemolysis and decreased colony size compared with strain MGAS5005 and strain comp $\Delta c c p A$. All three images are of same magnification with size indications at right. $(B)$ Changes in colony hemolysis and size were associated with altered pel/sag $A$ and has $A$ gene transcript levels. Strain MGAS5005 and $\triangle c c p A$ were grown in THY to early- and late-logarithmic growth phase, and pel/sagA and has $A$ transcript levels were measured by real-time TaqMan QRT-PCR with the $\Delta \Delta C_{T}$ method. Values above the $x$ axis indicate higher gene transcript levels in the $\triangle c c p A$ strain, whereas values below the $x$ axis indicate higher gene transcript levels in strain MGAS5005. Error bars indicate standard deviation among quadruplicate samples done on two separate occasions.

(data not shown). There was no significant difference in $\mathrm{pel} / \mathrm{sag} A$ or has $A$ transcript level between the wild-type and $\operatorname{comp} \Delta c c p A$ strains (data not shown). Therefore, we conclude that CcpA directly or indirectly influences the transcript levels of GAS genes encoding critical virulence factors.

Analysis of the CcpA Transcriptome. To test the hypothesis that CcpA influences a broader array of GAS genes, we next compared the genomewide transcriptome of wild-type strain MGAS5005 and the $\Delta c c p A$ isogenic mutant strain grown in THY. After accounting for multiple comparisons, in the earlyexponential growth phase the level of transcripts of $\approx 20 \%$ of all ORFs differed between the wild-type and $\Delta c c p A$ isogenic mutant strain (Fig. $2 A$, SI Table 3 ). In the late-exponential phase $\approx 10 \%$ of all ORFs were differentially expressed.

The largest number of transcripts differentially expressed between the two isogenic strains encode proteins known to be or putatively involved in carbohydrate transport and metabolism (Fig. 2B, SI Table 3). The differentially expressed genes included ATP-binding cassette (ABC) transporters or PTS operons responsible for glucose, lactose, maltodextrin, mannose, fructose, cellobiose, lactose, galactose, tagatose, and sialic acid transport. The transcript levels of genes in all of the aforementioned operons were increased in the $\Delta c c p A$ strain except for the maltodextrin operon. The decreased level of the maltodextrin 

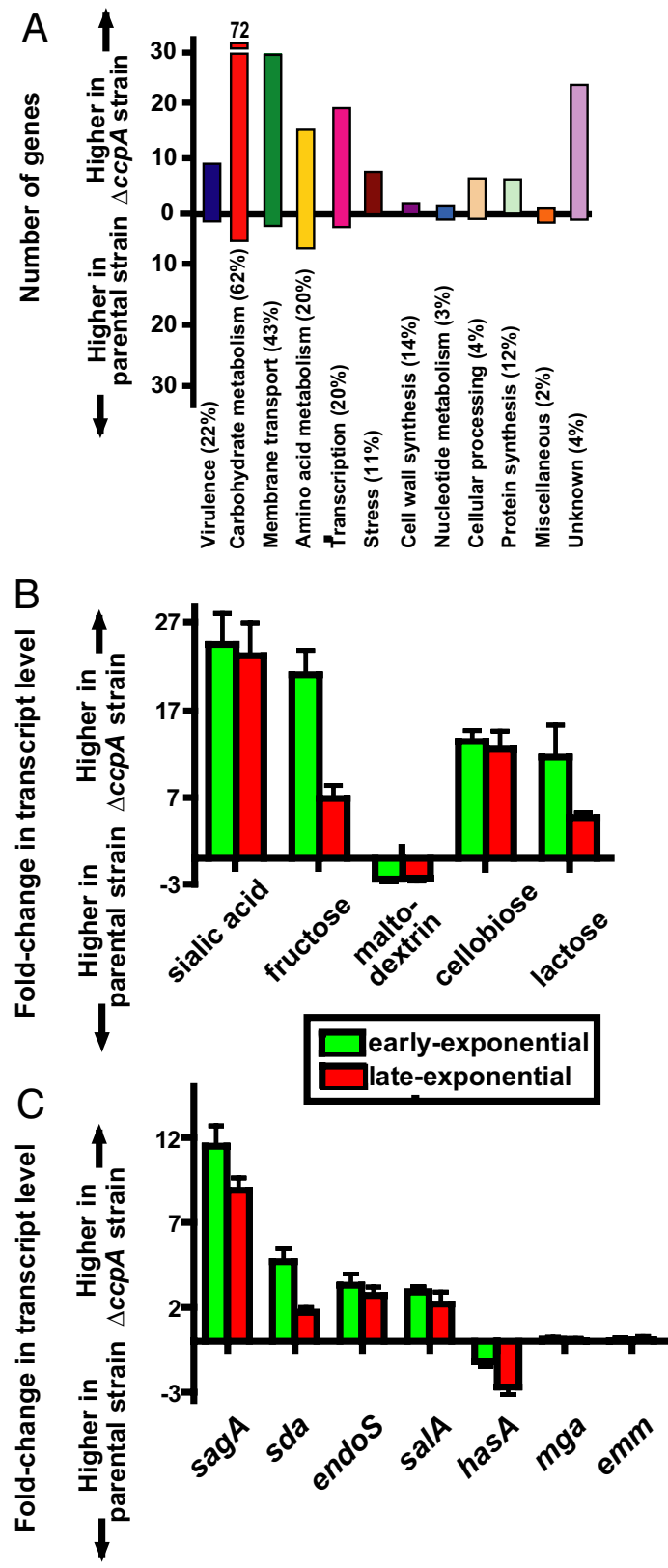

Fig. 2. Analysis of the CcpA transcriptome. Strain MGAS5005 and $\triangle \subset c p A$ were grown to early- and late-logarithmic growth phase in THY, and expression microarray analysis was performed as described in Materials and Methods. Values above the $x$ axis indicate higher gene transcript levels in the $\triangle \operatorname{ccp} A$ strain and values below the $x$ axis indicate higher gene transcript levels in wild-type strain MGAS5005. (A) Summary of genes with altered transcript levels grouped by functional category. Percentage numbers refer to genes affected by CcpA inactivation/total number of genes in that particular category in the entire GAS genome. $(B)$ Genes encoding carbohydrate utilization proteins. Shown are the mean fold changes in transcript levels of genes in an operon known or putatively involved in the transport and/or metabolism of the indicated carbohydrate. The transcript levels of all genes in the indicated operons were similarly affected. (C) Mean transcript levels of genes encoding select virulence factors. For $B$ and $C$, error bars show standard deviation among four samples.

operon in the $\Delta c c p A$ strain is in accord with the decreased growth of the $\triangle c c p A$ strain in a maltodextrin medium (SI Fig. 9C). We also observed significant differences in transcript levels of 21 genes encoding known and putative transcriptional regulators. Six of these genes comprise three two- component (TCS) gene regulatory systems, including M5005_spy0784/5, M5005_spy1305/6, and sptR/sptS. M5005_spy0784/5 positively influences a putative mannose/ fructose phosphotransferase system, whereas $s p t R / s p t S$ affects carbohydrate metabolism and virulence factor production in human saliva $(15,21)$.

A third major category of genes affected in the $\Delta c c p A$ mutant strain included those encoding proteins putatively or known to be involved in GAS virulence. As predicted from QRT-PCR data, the transcript levels of the nine-gene operon encoding streptolysin $\mathrm{S}$ were significantly elevated in the $\Delta c c p A$ strain, whereas the entire has $A B C$ operon involved in capsule synthesis was significantly down-regulated in the $\Delta c c p A$ strain (Fig. 2C). Other virulence factors affected by $\operatorname{cp} A$ inactivation included $s p d$, which encodes an extracellular DNase, and endoS, which encodes a protein that cleaves human $\operatorname{Ig}(22,23)$. No significant difference in transcript levels was observed for emm, which encodes the anti-opsonophagocytic M protein, or $m g a$, which encodes a transcriptional regulator involved in up-regulation of several virulence factors (Fig. 2C). Taken together, we conclude that $\mathrm{CcpA}$ is a global regulator of carbohydrate metabolism in GAS and has important effects on regulation of genes encoding transcriptional regulators and major virulence factors.

CcpA Contributes to GAS Virulence and Ability to Colonize the Mouse Oropharynx. In light of our findings that CcpA affects the transcript levels of multiple GAS virulence factors, we next tested the hypothesis that the $\Delta c c p A$ isogenic mutant strain was less virulent for mice than for the wild-type parental strain. Consistent with the hypothesis, significantly more mice inoculated i.p. with wild-type strain MGAS5005 died than with the $\triangle c c p A$ isogenic mutant strain $(P<0.001$; Fig. $3 A)$. Similarly, the comp $\Delta c c p A$ strain was significantly more virulent than the $\triangle c c p A$ strain $(P<0.001)$ and as virulent as strain MGAS5005 $(P=0.072)$.

Next, we tested the hypothesis that $\operatorname{cp} A$ contributes to the ability of GAS to colonize the mouse oropharynx. As hypothesized, after intranasal inoculation, the percentage of mice colonized over time with strain MGAS5005 was significantly greater than the $\Delta c c p A$ mutant strain $(P<0.001$; Fig. $3 B)$. Similarly, the average number of CFUs recovered from mice inoculated with strain MGAS5005 was significantly greater than with the $\Delta c c p A$ mutant strain $(P<0.001$; data not shown $)$. The comp $\Delta c c p A$ strain was recovered at significantly higher CFUs and from more mice compared with the $\Delta c c p A$ strain $(P<0.01$ for both). Taken together, these data indicate that $\mathrm{CcpA}$ contributes to GAS virulence and ability to colonize the mouse oropharynx.

CcpA Affects the Transcript Levels of GAS Virulence Factors During Growth in Human Saliva. We next sought to determine whether, under glucose-limiting conditions, CcpA influences the transcript levels of genes encoding virulence factors not influenced by CcpA in glucose-rich conditions (e.g., THY). To this end, we tested the transcript levels of several GAS virulence factors in strain MGAS5005 and the $\Delta c c p A$ strain during growth in human saliva, a glucose-poor medium. The transcript levels of speB (encoding a cysteine protease), mac (encoding an Ig-degrading enzyme), and spd3 (encoding a DNase) were significantly higher in strain MGAS5005 than in the $\Delta c c p A$ strain during growth in human saliva (Fig. 4). These data demonstrate that CcpA is needed for GAS to respond to human saliva by increasing transcript levels of virulence factors shown to affect the ability of GAS to persist in human saliva $(s p e B)$ and cause pharyngitis in a non-human primate model $(\operatorname{spd} 3)(23,24)$.

Recombinant GAS CcpA Binds to the Streptolysin S Promoter Region. When complexed with its coeffector HPr-Ser-46-P, CcpA functions by binding to cognate DNA sites (cre) in the promoter 

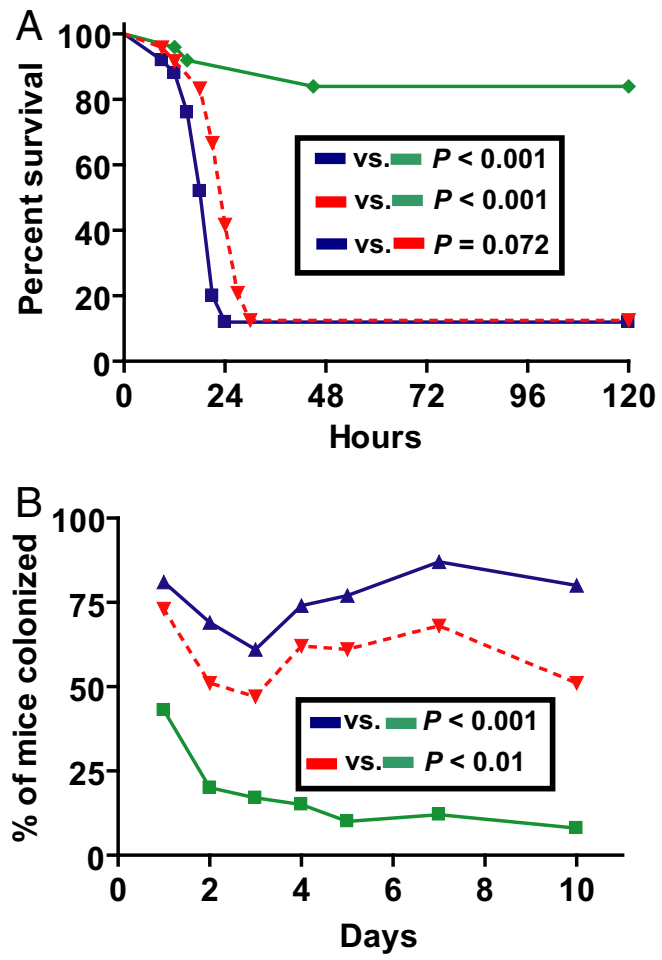

$\leftarrow$ MGAS5005 $\rightarrow-\Delta c c p A-\div-\operatorname{comp} \Delta c c p A$

Fig. 3. Inactivation of $\mathrm{CcpA}$ significantly decreases GAS virulence. (A) Invasive disease model. Twenty-five adult outbred CD-1 mice per group were inoculated i.p. with $\approx 1 \times 10^{7} \mathrm{CFU}$ of the indicated strains. Percent survival is graphed with $P$ values for Kaplan-Meier survival analysis. (B) Oropharyngeal colonization model. Adult outbred CD-1 mice (35 per group) were inoculated intranasally with $\approx 1.0 \times 10^{7} \mathrm{CFU}$ of the indicated strains. Mice oropharynxes were swabbed daily. Percentage of mice with GAS isolated by day with $P$ values shown for repeated measures analysis.

region or within target genes in $B$. subtilis $(25,26)$. A bioinformatic analysis of the genome of strain MGAS5005 identified 37 sites that contain a $B$. subtilis consensus cre sequence (TGWAANCGNTNWCA; see SI Text and SI Table 4). To elucidate whether CcpA exerts its regulatory effect on virulence factor production by direct DNA interaction, we analyzed the binding of purified recombinant GAS CcpA (SI Fig. 10) to DNA sequences in the promoter region of three different genes by fluorescence polarization: (i) pel/sagA, a virulence gene whose expression was repressed by CcpA in our transcriptome analysis; (ii) $l$ ct $O$, which contains a consensus cre sequence and was differentially transcribed between strain MGAS5005 and the $\Delta c c p A$ mutant strain and thus served as a positive control; and (iii) $f t s X$, a gene that does not contain a consensus cre sequence, was not differentially expressed in the transcriptome analysis, and therefore was chosen as a negative control (SI Table 5). GAS CcpA bound specifically to the pel/sagA DNA (Fig. 5). The binding affinity was specific $\left(K_{\mathrm{d}}=950 \pm 98 \mathrm{nM}\right.$, assuming a $100 \%$ active $\mathrm{CcpA}$ ) and enhanced $\approx 65$-fold by the presence of $50 \mu \mathrm{M}$ HPr-Ser-46-P $\left(K_{\mathrm{d}}=14.5 \pm 2.2 \mathrm{nM}\right)$, further supporting the notion that CcpA binds to the $\mathrm{pel} / \mathrm{sag} A$ promoter in vivo and controls its transcription. Nonphosphorylated HPr did not increase the CcpA-DNA interaction (data not shown). Similar results were found for $l c t O$, and, as expected, specific binding was not observed for the promoter region of $f t S X$ (SI Fig. 11). Thus, binding of CcpA to the promoter region of $\mathrm{pel} / \mathrm{sag} A$ demonstrates a direct mechanism of $\mathrm{CcpA}$ regulation of a key GAS virulence factor.

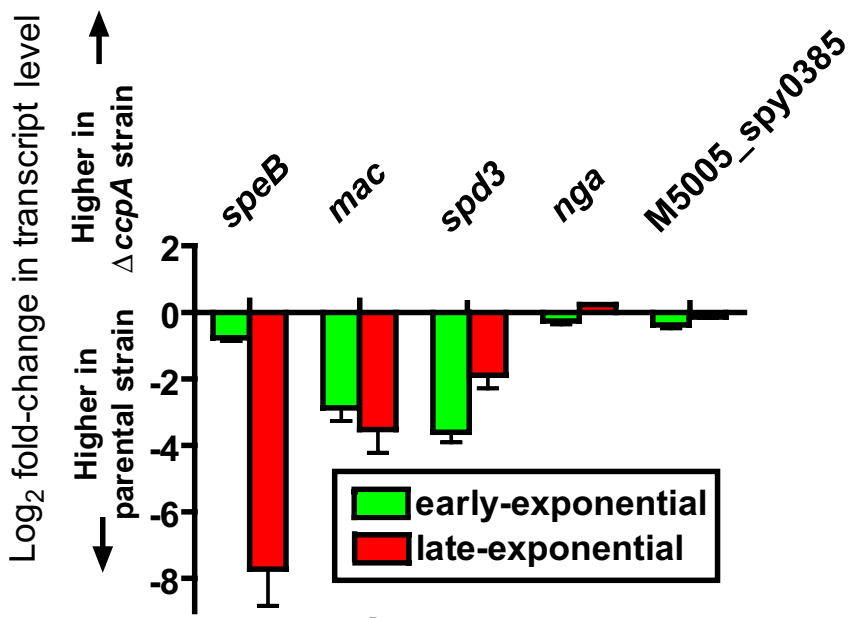

Fig. 4. $\mathrm{CcpA}$ affects GAS virulence factor production in glucose-limited medium. Gene transcript levels were measured by TaqMan real-time QRT-PCR in strains MGAS5005 and $\triangle c c p A$ grown to early- and late-logarithmic growth phase in human saliva. Bars indicate $\log _{2}$ differences in gene transcript levels between the two strains with error bars showing standard deviation for quadruplicate samples done on two separate occasions. Values above the $x$ axis indicate higher gene transcript levels in the $\triangle c c p A$ strain and values below the $x$ axis indicate higher gene transcript levels in strain MGAS5005.

\section{Discussion}

Although CcpA orthologs have been investigated in other Grampositive organisms, before this study the CcpA transcriptome had not been determined in a human pathogen $(8,9,27,28)$. A key discovery was that CcpA influenced the transcript levels of several GAS virulence factors, including the potent cytolysin streptolysin S, the extracellular DNase Spd, and the Ig-degrading EndoS. In Bacillus spp., transport of glucose or other readily metabolized carbohydrates through the PTS system leads to phosphorylation of the phosphocarrier protein HPr at residue Ser-46, resulting in HPr-Ser-46-P (6). HPr-Ser-46-P serves as a

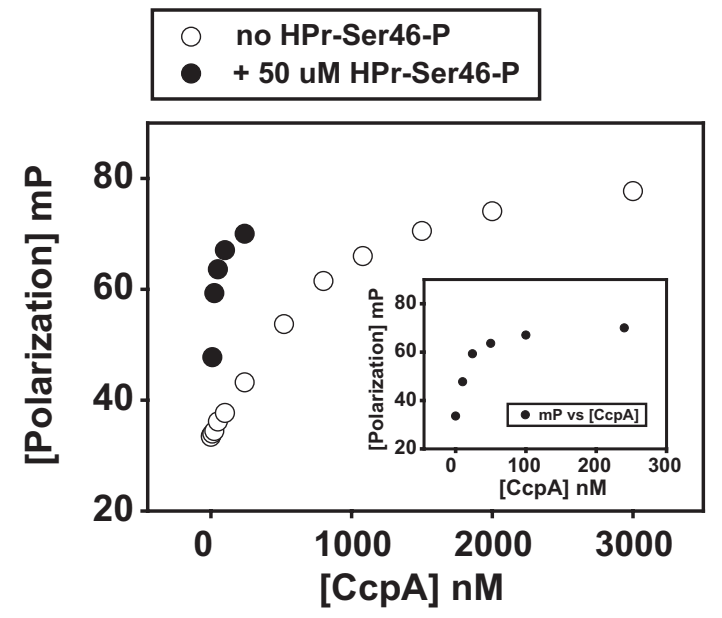

Fig. 5. The CcpA-(HPr-Ser-46-P) complex interacts specifically with the promoter region of pel/sagA. Purified GAS CcpA was titrated to $1 \mathrm{nM}$ pel/sagA cre in the absence (open circles) and presence (closed circles) of $50 \mu \mathrm{M}$ HPr-Ser-46-P. Millipolarization units (mP) are plotted against the CcpA concentration. The shift to the left in the binding curve demonstrates stimulation of DNA-CcpA complex formation by HPr-Ser-46P ( $K_{\mathrm{D}}$ f for DNA interaction are $948 \pm 89 \mathrm{nM}$ and $14.5 \pm 2.2 \mathrm{nM}$ with and without HPr-Ser-46P, respectively). (Inset) Shown is relationship between CcpA concentration and $\mathrm{mP}$ units in the presence of HPr-Ser-46-P to clarify that saturation is already reached at a CcpA concentration of $240 \mathrm{nM}$. 


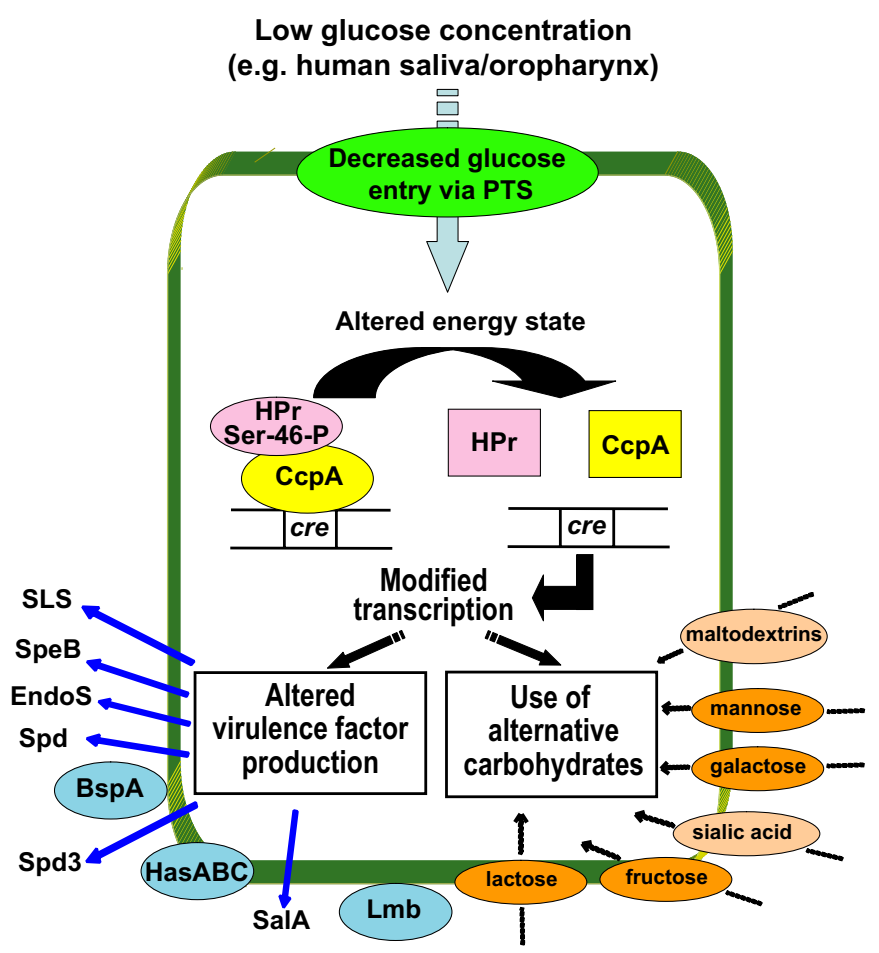

Fig. 6. Hypothesis explaining how CcpA affects GAS carbohydrate utilization and virulence factor production. Decreased extracellular glucose levels result in dephosphorylation of HPr-Ser-46-P leading to dissociation of HPr and CcpA. $\mathrm{CcpA} / \mathrm{HPr}$ dissociation results in changes in CcpA binding to DNA catabolite response element sites (cre) and altered transcription. Systems involved in the uptake and utilization of specific carbohydrates are shown on the right side of the figure. Phosphotransferase systems are shown in orange and ATP-binding cassette transport systems are in tan. On the left side of the figure, in blue, are virulence factors with the relationship of the protein names to the cell surface corresponding to their inferred location (e.g., actively secreted, anchored to the cell wall, or embedded in the cell membrane). Putative or known functions of affected virulence factors: SLS, cytolysin; SpeB, cysteine protease; EndoS, Ig cleavage; Spd and Spd3, DNases; BspA, epithelial cell-binding protein; Has$A B C$, hyaluronic acid capsule production; SalA, salivaricin production; Lmb, laminin binding protein.

protein coeffector for $\mathrm{CcpA}$ that mediates $\mathrm{CcpA}$ binding to $\mathrm{cre}$ sites, which ultimately results in altered gene expression (26). Strain MGAS5005 contains a gene (M5005_spy1121) with 66\% identity and $82 \%$ similarity at the amino acid level to HPr in Bacillus subtilis subsp. subtilis strain 168, suggesting that similar CcpA pathways are likely to function in GAS. Our GAS data suggest a model that, when environmental glucose levels are low, such as in the human oropharynx and saliva, CcpA-(HPr-Ser46-P)-cre interaction is relieved, thus allowing transcription of carbohydrate utilization genes and virulence factors vital to host-pathogen interaction (Fig. 6).

Although CcpA has been shown to influence virulence factor production in other Gram-positive pathogens, there is no information available regarding the mechanism by which this occurs $(8,27,28)$. Our finding that CcpA binds to the $\mathrm{pel} / \mathrm{sag} A$ promoter region demonstrates a direct link in GAS between environmental carbohydrate concentrations and virulence factor production. In light of the known lytic effect of streptolysin S on neutrophils, the data suggest that the nutritional status of GAS may serve as a mechanism for sensing when key virulence factors are needed to inhibit host defenses (29). Alternatively, streptolysin S may have a unappreciated role in nutrient acquisition. Because sag $A$ is transcribed as part of the larger regulatory RNA pel, our data demonstrating that CcpA affects transcript levels of an RNA regulatory system are in accordance with a study in Staphylococcus aureus in which CcpA influenced transcript levels of $R N A I I$, the RNA effector molecule of the $\operatorname{agr}$ system $(8,20)$. Taken together, our findings substantiate the idea that in Gram-positive pathogens CcpA interacts with RNA regulatory systems, the importance of which are being increasingly appreciated in both humans and microbes.

Finally, we also discovered that CcpA positively influences the transcript level of several virulence factors, including speB, during growth in the nutrient-limited medium of human saliva. Given that human saliva is the first substance with which GAS interacts in the oropharynx, these data strongly suggest that GAS $\mathrm{CcpA}$ is required for the up-regulation of key virulence factors during the initial stages of host-pathogen interaction (24). The absence of increased production of key virulence factors early during pharyngeal infection may explain the relative inability of the $\Delta c c p A$ mutant strain to colonize the oropharynx. Thus, our data demonstrate the GAS CcpA directly represses production of virulence factors, such as streptolysin S, under nutrient-rich conditions and augments production of other virulence factors, such as SpeB, under nutrient-limited conditions, thereby providing a key mechanism by which GAS responds to changing environments (Fig. 6).

Human pathogenic microbes differentially regulate production of key virulence factors in vivo, a hallmark of pathogen-host interaction. We have discovered that the major human pathogen GAS modulates virulence factor production required for survival and infectivity by a CcpA-mediated pathway. Given the highly conserved nature of CcpA, similar genomewide studies of other Gram-positive pathogens may yield enhanced understanding of links between basic metabolic processes and pathogenesis.

\section{Materials and Methods}

Bacterial Strains and Culture Media. Serotype M1 strain MGAS5005 is genetically representative of the clone responsible for most contemporary (post1987) human infections; its genome has been sequenced (30). The $\triangle$ ccpA isogenic mutant strain was created from parental serotype M1 strain MGAS5005 by nonpolar insertional mutagenesis. We used pDC123, a plasmid capable of replicating in GAS, to genetically complement the $\triangle$ ccpA strain in trans to create the comp $\Delta c c p A$ strain (SI Text). Ultrapure carbohydrates (Sigma) were added at a concentration of $0.5 \%(\mathrm{wt} / \mathrm{vol})$ to a carbohydratefree preparation of a commercially available, chemically defined medium (CDM; SAFC Biosciences) to create glucose medium, maltose medium, etc. Growth in human saliva was performed with specimens collected from healthy adult volunteers under a protocol approved by the Baylor College of Medicine Institutional Review Board (24).

RNA Isolation, TaqMan Transcript Level Analysis, and Expression Microarray Analysis. RNA was purified from a minimum of four replicate cultures by using an RNeasy kit (Qiagen). The concentration and quality of RNA were assessed with an Agilent 2100 Bioanalyzer and analysis of the $A_{260} / A_{280}$ ratio. Select gene transcript level analysis was performed with TaqMan real-time QRT-PCR (primers and probes listed in SI Table 6). A custom-made Affymetrix GeneChip that contains $100 \%$ of the ORFs of strain MGAS5005 was used for expression microarray (transcriptome) studies. Principal component analysis (PCA) indicated that the data were of high quality and that the two time points provided distinct information regarding GAS gene transcripts differentially expressed between these two strains (SI Fig. 12). To compare gene transcript levels between the wild-type and mutant strain, a two-sample $t$ test (unequal variance) was applied, followed by a false discovery rate correction $(Q<0.05)$ to account for multiple testing. Genes were considered differentially transcribed if the $t$ test had a corrected $P$ value of $<0.05$ and the difference in mean gene transcript level was at least 2 -fold. For further information on assessment of transcript levels see SI Text.

Mouse Virulence Studies. Mouse experiments were performed according to protocols approved by the Baylor College of Medicine Institutional Animal Care and Use Committee. For the invasive disease model, 25 female outbred CD-1 Swiss mice (Harlan-Sprague-Dawley) were injected i.p. with $\approx 2.5 \times 10^{7}$ GAS CFU (31). Throat colonization studies were performed by inoculating 35 mice in each group with $1 \times 10^{7} \mathrm{CFU}$ of GAS (32). The throat of each mouse was swabbed before inoculation and then daily thereafter. 
Purification and Binding Characteristics of CсpA. СсpA was purified to homogeneity from Escherichia coli (SI Fig. 10, SI Text). Fluorescence anisotropy was used to determine the binding characteristics of CcpA to 5'-fluoresceinlabeled oligonucleotides in the presence and absence of $50 \mu \mathrm{M}$ HPr-Ser-46-P (33, 34; SI Text).

1. Loughman JA, Caparon MG (2006) A novel adaptation of aldolase regulates virulence in Streptococcus pyogenes. EMBO J 25:5414-5422.

2. Moyrand F, Fontaine T, Janbon G (2007) Systematic capsule gene disruption reveals the central role of galactose metabolism on Cryptococcus neoformans virulence. Mol Microbiol 64:771-781.

3. Larocque RC, et al. (2005) Transcriptional profiling of Vibrio cholerae recovered directly from patient specimens during early and late stages of human infection. Infect Immun 73:4488-4493.

4. Faucher SP, Porwollik S, Dozois CM, McClelland M, Daigle F (2006) Transcriptome of Salmonella enterica serovar Typhi within macrophages revealed through the selective capture of transcribed sequences. Proc Natl Acad Sci USA 103:1906-1911.

5. Moreno MS, Schneider BL, Maile RR, Weyler W, Saier MH, Jr (2001) Catabolite repression mediated by the CcpA protein in Bacillus subtilis: novel modes of regulation revealed by whole-genome analyses. Mol Microbiol 39:1366-1381.

6. Deutscher J, Francke C, Postma PW (2006) How phosphotransferase system-related protein phosphorylation regulates carbohydrate metabolism in bacteria. Microbiol Mol Biol Rev 70:939-1031.

7. Henkin TM, Grundy FJ, Nicholson WL, Chambliss GH (1991) Catabolite repression of alpha-amylase gene expression in Bacillus subtilis involves a trans-acting gene product homologous to the Escherichia coli lacl and galR repressors. Mol Microbiol 5:575-584.

8. Seidl K, et al. (2006) Staphylococcus aureus CcpA affects virulence determinant production and antibiotic resistance. Antimicrob Agents Chemother 50:1183-1194.

9. Iyer R, Baliga NS, Camilli A (2005) Catabolite control protein A (CcpA) contributes to virulence and regulation of sugar metabolism in Streptococcus pneumoniae. $J$ Bacteriol 187:8340-8349.

10. Iyer R, Camilli A (2007) Sucrose metabolism contributes to in vivo fitness of Streptococcus pneumoniae. Mol Microbiol 66:1-13.

11. Sonenshein AL (2007) Control of key metabolic intersections in Bacillus subtilis. Nat Rev Microbiol 5:917-927.

12. Tart AH, Walker MJ, Musser JM (2007) New understanding of the group A Streptococcus pathogenesis cycle. Trends Microbiol 15:318-325.

13. Kreikemeyer B, Mclver KS, Podbielski A (2003) Virulence factor regulation and regulatory networks in Streptococcus pyogenes and their impact on pathogen-host interactions. Trends Microbiol 11:224-232.

14. Malke H, Steiner K, McShan WM, Ferretti JJ (2006) Linking the nutritional status of Streptococcus pyogenes to alteration of transcriptional gene expression: The action of CodY and RelA. Int J Med Microbiol 296:259-275.

15. Shelburne SA, et al. (2005) Central role of a two-component gene regulatory system of previously unknown function in pathogen persistence in human saliva. Proc Natl Acad Sci USA 102:16037-16042.

16. Cho KH, Caparon MG (2005) Patterns of virulence gene expression differ between biofilm and tissue communities of Streptococcus pyogenes. Mol Microbiol 57:15451556 .
ACKNOWLEDGMENTS. We thank B. Lei for providing the vector used to overexpress CcpA. This work was supported by American Heart Association Grant 0565133Y (to S.A.S), National Institute of Allergy and Infectious Diseases K08 Career Development Award AI-064564 (to S.A.S.), and funds from the Robert A Welch Foundation G-0040 (R.G.B).

17. Virtaneva K, et al. (2005) Longitudinal analysis of the group A Streptococcus transcrip tome in experimental pharyngitis in cynomolgus macaques. Proc Natl Acad Sci USA 102:9014-9019.

18. Wessels MR, Moses AE, Goldberg JB, DiCesare TJ (1991) Hyaluronic acid capsule is a virulence factor for mucoid group A streptococci. Proc Natl Acad Sci USA 88:8317-8321.

19. Nizet V, et al. (2000) Genetic locus for streptolysin S production by group A Streptococcus. Infect Immun 68:4245-4254.

20. Mangold M, et al. (2004) Synthesis of group A streptococcal virulence factors is controlled by a regulatory RNA molecule. Mol Microbiol 53:1515-1527.

21. Sitkiewicz I, Musser JM (2006) Expression microarray and mouse virulence analysis of four conserved two-component gene regulatory systems in group A Streptococcus. Infect Immun 74:1339-1351.

22. Collin M, Olsen A (2001) EndoS, a novel secreted protein from Streptococcus pyogenes with endoglycosidase activity on human IgG. EMBO J 20:3046-3055.

23. Sumby P, et al. (2005) Extracellular deoxyribonuclease made by group A Streptococcus assists pathogenesis by enhancing evasion of the innate immune response. Proc Nat Acad Sci USA 102:1679-1684.

24. Shelburne SA, III, et al. (2005) Growth characteristics of and virulence factor production by group A Streptococcus during cultivation in human saliva. Infect Immun 73:4723-4731.

25. Warner JB, Lolkema JS (2003) CcpA-dependent carbon catabolite repression in bacteria. Microbiol Mol Biol Rev 67:475-490.

26. Schumacher MA, et al. (2004) Structural basis for allosteric control of the transcription regulator CcpA by the phosphoprotein HPr-Ser46-P. Cell 118:731-741.

27. Giammarinaro P, Paton JC (2002) Role of RegM, a homologue of the catabolite repressor protein $\mathrm{CcpA}$, in the virulence of Streptococcus pneumoniae. Infect Immun 70:5454-5461.

28. Varga J, Stirewalt VL, Melville SB (2004) The CcpA protein is necessary for efficient sporulation and enterotoxin gene (cpe) regulation in Clostridium perfringens. J Bacteriol 186:5221-5229.

29. Miyoshi-Akiyama T, et al. (2005) Cytocidal effect of Streptococcus pyogenes on mouse neutrophils in vivo and the critical role of streptolysin S. J Infect Dis 192:107-116.

30. Sumby P, et al. (2005) Evolutionary origin and emergence of a highly successful clone of serotype M1 group A Streptococcus involved multiple horizontal gene transfer events. J Infect Dis 192:771-782.

31. Sumby P, Whitney AR, Graviss EA, DeLeo FR, Musser JM (2006) Genome-wide analysis of group a streptococci reveals a mutation that modulates global phenotype and disease specificity. PLoS Pathog 2:e5.

32. Shelburne SA, III, et al. (2006) Maltodextrin utilization plays a key role in the ability of group A Streptococcus to colonize the oropharynx. Infect Immun 74:4605-4614.

33. Kliegman Jl, Griner SL, Helmann JD, Brennan RG, Glasfeld A (2006) Structural basis for the metal-selective activation of the manganese transport regulator of Bacillus subtilis. Biochemistry 45:3493-3505.

34. Lundblad JR, Laurance M, Goodman RH (1996) Fluorescence polarization analysis of protein-DNA and protein-protein interactions. Mol Endocrinol 10:607-612. 